\title{
Study of New Criteria Based on Eigenvalue Decomposition to Assist Arrhythmogenic Cardiomyopathy Diagnosis
}

\author{
Santiago Jiménez-Serrano ${ }^{1}$, Jorge Sanz Sánchez ${ }^{2}$, Antonio Cebrián ${ }^{1}$, Begoña Igual $^{3}$, \\ Raquel Cervigón ${ }^{4}$, José Millet ${ }^{1}$, Esther Zorio ${ }^{2}$, Francisco Castells ${ }^{1}$ \\ ${ }^{1}$ Instituto ITACA, Universitat Politècnica de València, València, Spain \\ ${ }^{2}$ Hospital Universitario y Politécnico La Fe, València, Spain \\ ${ }^{3}$ Centro Médico-Quirúrgico ERESA, València, Spain \\ ${ }^{4}$ Universidad de Castilla-La Mancha, Cuenca, Spain
}

\begin{abstract}
Arrhythmogenic Cardiomyopathy (ACM) is a heritable cardiac disease causing sudden cardiac death in young people. Its clinical diagnosis includes major and minor criteria based on alterations of the electrocardiogram (ECG). The aim of this study is to propose and evaluate new criteria based on Principal Component Analysis (PCA) applied to 12-lead ECG signals and find differences between individuals affected and unaffected by ACM.

Datasets consist of 12-lead ECG recordings from 32 patients diagnosed with ACM, and 37 relatives of those affected, but without gene mutation. We extracted 8 eigenvalues from each recording. Next, we proposed two criteria (C1 and $\mathrm{C} 2$ ) representing the percentage of energy in eigenvalues of non-dipolar and third principal components respectively.

Results show that the most significant indicator was $C 1$, with values of $0.026 \pm 0.015$ in ACM patients and $0.014 \pm 0.009$ in those not affected by ACM $(p<10 e-3)$. C2 has values of $0.050 \pm 0.035$ and $0.028 \pm 0.023(p<10 e-2)$ in the same groups. The results of this study show significant differences in the indicators proposed between those affected by ACM and the control group, indicating that the degree of information contained in the nondipolar leads is higher in the first group. All the above suggest an increased heterogeneity of the electrical activation in patients with ACM.
\end{abstract}

\section{Introduction}

Arrhythmogenic Cardiomyopathy (ACM) is a heritable cardiac disease that causes fibro-fatty infiltration in the myocardial tissue [1], producing electrical conduction disturbances that can trigger ventricular arrhythmias.
ACM is one of the leading causes of sudden death in young people from heart failure with dominant transmission. The chances of getting the disease increases dramatically among relatives of those affected [2, 3].

The clinical diagnosis of ACM is complicated and is based on the Task Force criteria reviewed in 2010 [4], mainly based on previous studies with predominant involvement of the right ventricle, but still not very sensitive to diagnose the predominant forms in the left ventricle. These criteria include observations on the surface electrocardiogram (ECG), Magnetic Resonance Imaging (MRI), echocardiography, prevalence among relatives and histological analysis.

Regarding these diagnostic criteria, the standard ECG is the simplest and most commonly used technique for detecting cardiac arrhythmias. Certain ECG morphological changes have been identified as indicative of possible ACM. Among others, T-wave inversion in the right precordial leads (V1-V3), parameters related to the duration, amplitude, and fragmentation of the QRS complex along with epsilon wave appearance in different derivations have been described [4-6]. However, other indicators that have proven ECG diagnostic efficacy in other diseases of ventricular origin have not been tested in ACM.

On the other hand, Principal Component Analysis (PCA) has been successfully used in ECG signal processing in different applications [7, 8].

The aim of this study is to evaluate new criteria based on eigenvalue decomposition corresponding to 12-lead ECG registers and look for differences in behaviour among subjects affected by ACM and those who do not suffer the disease. The initial hypothesis is that differences exist in the relationship among eigenvalues extracted from PCA analysis of ECG registers between these two groups. This paper presents a set of indicators defined from eigenvalue decomposition in order to extract and quantify this information. 


\section{Materials}

As database for this study we included records from 32 patients previously diagnosed with ACM (23 with preferential involvement of the left ventricle, and 9 with biventricular involvement). Furthermore, as control group we used records from 37 subjects who had undergone diagnostic tests for ACM due to having relatives affected by the disease, but with negative results in their own diagnosis without any finding of ACM gene mutation. Each of these registers consists of ECG signals of 12 standard leads, lasting 10 to 15 seconds with sampling frequency of $500 \mathrm{~Hz}$, acquired through a Philips PageWriter Touch device.

\section{Methods}

This section describes the methods to extract and quantify the proposed parameters based on eigenvalues decomposition from the standard 12-leads ECG recordings. This includes signal preprocessing, PCA, parameter definition and statistical analysis. All these stages were carried on through MATLAB (2014b, The MathWorks).

\subsection{Signal preprocessing}

First, we applied a $50 \mathrm{~Hz}$ notch filter in order to remove the power line interference. Next, we applied a band-pass filter between $1 \mathrm{~Hz}$ and $45 \mathrm{~Hz}$ in order to reduce high frequency noise and baseline wandering. Finally, we removed the first and last 0.5 seconds of signal with the aim of avoiding the transient filtering stage.

\subsection{PCA}

PCA is a multivariate statistical technique that allows dimensionality reduction of large correlated data sets into another data set of lower dimensions. Each of these new dimensions is called "principal components" and can represent better the original data variance [9].

Once signals were filtered we performed a PCA from each recording. Data from 12 leads were organized in a matrix of 12 rows and $n$ columns, being $n$ the number of samples for each filtered recording. Next, we performed a Singular Value Decomposition (SVD) of the data matrix. Since PCA transforms the ECG to virtual parameters that are mutually independent (i.e. orthogonal), the 3 first principal components contain all the information representing the vectorial concept of a single electrical dipole. The remaining principal components represent the non-dipolar components in the ECG, in this case from the fourth to the eighth. Finally, in order to quantify the variance in dipolar and non-dipolar components we extracted the first 8 eigenvalues.

\subsection{Parameter definitions}

Each eigenvalue represents the variance for each principal component corresponding to the 12-leads recording for each subject. The sum of the 8 eigenvalues represents the $100 \%$ of the variance in the principal components. First eigenvalue contains the highest variance since corresponds to the first and most important principal component. On the other hand, the eighth eigenvalue contains the lowest variance. From the definitions above, we proposed the following two criteria in order to assist ACM diagnosis.

The first criteria (C1) represents the percentage of variance in eigenvectors in non-dipolar components and is defined as:

$$
C 1=\frac{\sum_{i=4}^{8} \text { eigenvalue }_{i}}{\sum_{i=1}^{8} \text { eigenvalue }_{i}}
$$

That is, the sum of variance from the fourth to the eighth eigenvalue divided by the whole variance contained at the eight components. This gives us a measure of energy of the variance contained in the nondipolar components. Values of this criteria are defined in the interval $(0,1)$, showing the lower bound lack of variance in the non-dipolar components.

The second proposed criteria (C2) represents the level of energy contained at the third eigenvalue among the dipolar components and is defined as:

$$
C 2=\frac{\text { eigenvalue }_{3}}{\sum_{i=1}^{2} \text { eigenvalue }_{i}}
$$

This metric explains the variance of energy among the dipolar components. As before, C2 values are defined in the interval $(0,1)$, showing the lower bound lack of variance in the third dipolar component. C2 indicates the level of contribution of the dipole activation in the third dimension. In case of a very low value, activation could be explained from a two-dimensional space (i.e. a plane).

\subsection{Statistical analysis}

After obtaining the parameter values, results were compared in both groups of subjects in order to find statistically significant differences by Student's $t$ test. Previously, we used Kolmogorov-Smirnov test to corroborate normality in the data.

\section{Results}

Results show that the most significant indicator was C1, with values of $0.026 \pm 0.015$ in ACM patients and $0.014 \pm 0.009$ in not affected by ACM individuals $(\mathrm{p}<10 \mathrm{e}-$ 
3). This indicates that more percentage of variance is contained in the non-dipolar components among the ACM patients. On the other hand, within the control group, non-dipolar components contain less information since $\mathrm{C} 1$ values are lower.

Second criteria C2, contains values of $0.050 \pm 0.035$ and $0.028 \pm 0.023(\mathrm{p}<10 \mathrm{e}-2)$ in the same group division. C2 values show a highest percentage of variance in the third eigenvalue among the dipolar components in the ACM patients. Table 1 summarize these results.

Table 1. Results of statistical analysis. Parameter values obtained (mean \pm standard deviation) and $\mathrm{p}$ value.

\begin{tabular}{llll}
\hline & $\begin{array}{l}\text { ACM Patients } \\
\mathrm{n}=32\end{array}$ & $\begin{array}{l}\text { Control Group } \\
\mathrm{n}=37\end{array}$ & $p$-value \\
\hline C1 & $0.026 \pm 0.015$ & $0.014 \pm 0.009$ & $1.13 \mathrm{e}-4$ \\
$\mathrm{C} 2$ & $0.050 \pm 0.035$ & $0.028 \pm 0.023$ & $2.98 \mathrm{e}-2$ \\
\hline
\end{tabular}

Figures 1 and 2 show these results in boxplots, presenting the values differences in median, mean, maximum and standard deviation in C1 and C2 between the ACM patients and the control group.

C1 and C2 results present higher and more heterogeneous values in data belonging to ACM patients, indicating that more variability is explained by the last 6 eigenvalues in presence of the disease. On the other hand, the first two eigenvalues could explain the most of the variance in the case of the control group since C1 and C2 values are closer and more concentrated to zero. Figure 3 shows a scatter plot presenting the dispersion of $\mathrm{C} 1$ and $\mathrm{C} 2$ values between ACM patients and the control group.

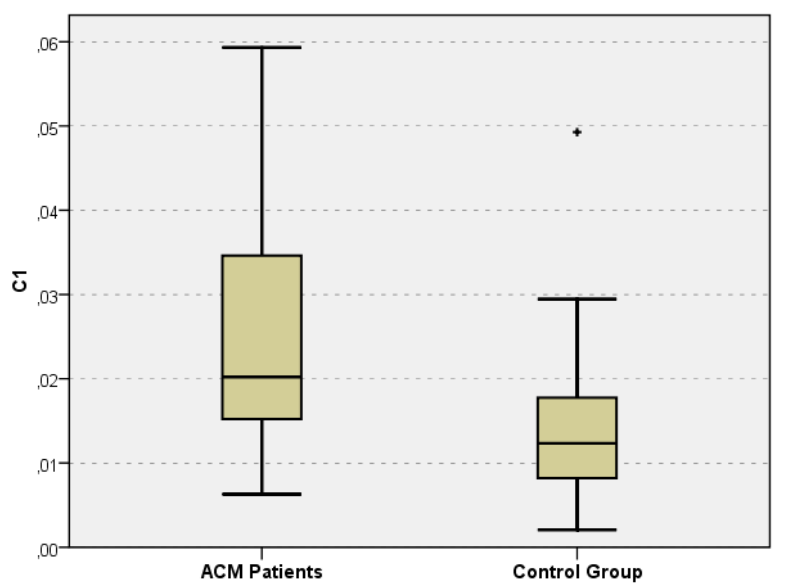

Figure 1: Boxplot obtained from C1 criteria values in the ACM patients $(n=32)$ and control group $(n=37)$.

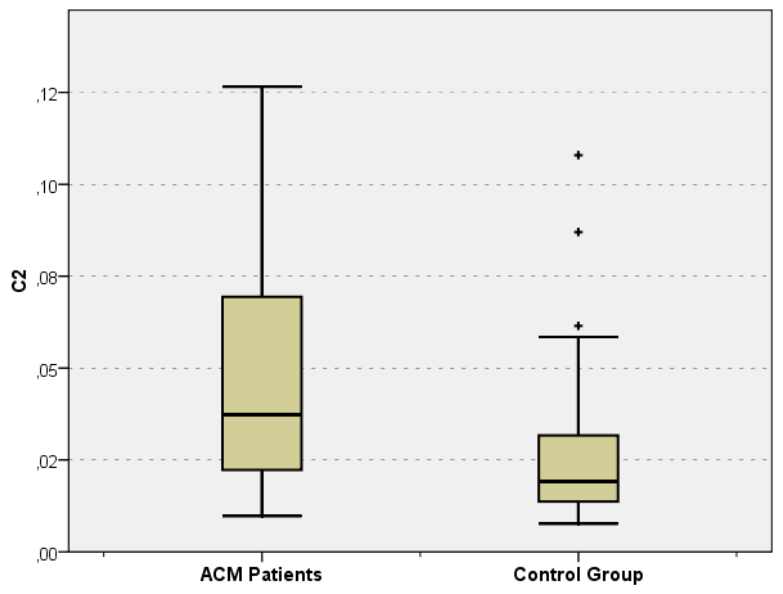

Figure 2: Boxplot obtained from C2 criteria values in the ACM patients $(n=32)$ and control group $(n=37)$.

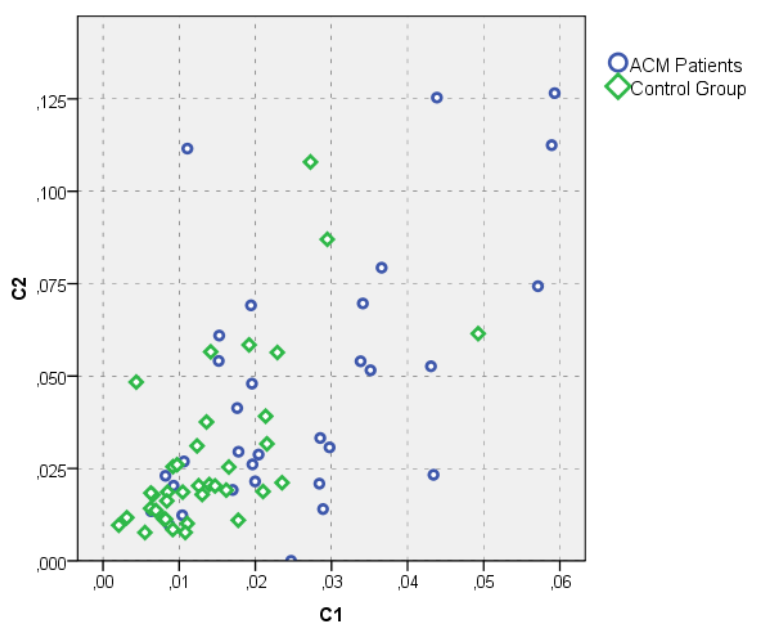

Figure 3: Scatter plot obtained from the values of $\mathrm{C} 1(\mathrm{x}-$ axis) and C2 (y-axis) parameters in the ACM patients $(n=32)$ and control group $(n=37)$.

\section{Discussion}

The main finding of this study is that criteria based on eigenvalue decomposition could assist to ACM diagnosis or characterization. This is especially relevant given that the control group studied during this work is composed by relatives of subjects affected by ACM. Hence the proposed criteria could be used in family medical screenings.

As expected, this study shows significant differences in the proposed criteria between the group of patients affected by ACM and the control group, being the variance in the eigenvalues of non-dipolar components higher in the first group. This has been empirically 
demonstrated by differences in groups with the proposed set of parameters. These findings are consistent with the ECG Task Force diagnostic criteria, since fragmentation of QRS or epsilon wave among others suggest a higher level of heterogeneity of the electrical activation in ACM patients [4-6]. However, it would be advisable to use a combination of parameters to improve these results. Thus it could increase sensitivity in the detection of MCA by ECG, as well as the degree of involvement.

Despite the results, this study has some limitations that should be considered for future works. First, the database of ECG records should be enlarged by including new patients and controls in order to gain statistical significance. Finally, although it has been possible to characterize the behavior of PCA and eigenvalues in the ECG recordings for both groups, the proposed methodology is not able to classify with no errors, subjects belonging to one group or another. Therefore, further and deeper research to improve these results is still needed.

\section{Conclusion}

In this paper we have proposed two new indicators in order to assist the ACM diagnostic based on eigenvalue decomposition of the ECG. The results of this study show significant differences in the indicators proposed between those affected by ACM and the control group, indicating that the degree of information contained in the nondipolar leads is higher in the first group. Besides, the level of information contained at the third dimension is also higher in the ACM group. All the above suggest an increased heterogeneity of the electrical activation in patients with ACM. These results could help to characterize the disease in a non-invasive way, especially within the context of family screening, improving sensitivity in detection by ECG.

\section{Acknowledgements}

This work was partially supported by Instituto de Salud Carlos III-FEDER "Una manera de hacer Europa" (PI14/014077 and RD12/0042/0029), National Program of the Spanish Ministry of Economy and Competitiveness (DPI2015-70821-R), ERESA 2013 grant and IIS La Fe-UPV 2014 grant.

\section{References}

[1] Marcus FI, Fontaine GH, Guiraudon G, Frank R, Laurenceau JL, Malerque C, Grosgogeat Y. Right ventricular dysplasia: a report of 24 adult cases. Circulation 1982; 65(2):384-98 (ISSN: 0009-7322)

[2] Nava A, Thiene G, Canciani B, Scognamiglio R, Daliento L, Buja G, Martini B, Stritoni P, Fasoli G. Familial occurrence of right ventricular dysplasia: a study involving nine families. Journal of the American College of Cardiology 1988; 12(5):1222-1228 (ISSN: 0735-1097).

[3] Daliento L, Turrini P, Nava A, Rizzoli G, Angelini A, Buja G, Scognamiglio R, Thiene G. Arrhythmogenic right ventricular cardiomyopathy in young versus adult patients: similarities and differences. Journal of the American College of Cardiology 1995; 25(3):655-664 (ISSN: 07351097).

[4] Marcus FI, McKenna WJ, Sherrill D, Basso C, Bauce B, Bluemke DA, Calkins H, Corrado D, Cox MG, Daubert JP, Fontaine G, Gear K, Hauer R, Nava A, Picard MH, Protonotarios N, Saffitz JE, Sanborn DM, Steinberg JS, Tandri H, Thiene G, Towbin JA, Tsatsopoulou A, Wichter T, Zareba W. Diagnosis of Arrhythmogenic Right Ventricular Cardiomyopathy/Dysplasia: proposed modification of the task force criteria. Circulation 2010; 121(3): 1533-1541 (ISSN: 0009-7322).

[5] Quarta G, Elliot PM. Criteria for Arrhythmogenic Right Ventricular Cardiomyopathy. Revista Española de Cardiología (English Version) 2012; 65(07): 599-605 (ISSN: 1885-5857).

[6] Pilichou K, Thiene G, Bauce B, Rigato I, Lazzarini E, Migliore F, Marra MP, Rizzo S, Zorzi Z, Daliento L, Corrado D, Basso C. Arrhythmogenic cardiomyopathy. Orphanet Journal of Rare Diseases 2016; 11(1):1-17 (ISSN: 1750-1172)

[7] Castells F, Laguna P, Sörnmo L, Bollmann A, Millet J. Principal Component Analysis in ECG Signal Processing. EURASIP Journal on Advances in Signal Processing 2007; 1:98-119 (ISSN: 1110-8657)

[8] Bonizzi P, Guillem MS, Climent AM, Millet J, Zarzoso V, Castells F, Meste $\mathrm{O}$. Noninvasive assessment of the complexity and stationarity of the atrial wavefront patterns during atrial fibrillation. IEEE Transactions on Biomedical Engineering 2010; 57(9):2147-2157 (ISSN: 0018-9294)

[9] Joliffe IT. Principal Component Analysis. New York, NY, USA: Springer, 2002.

Address for correspondence.

Santiago Jiménez Serrano

Universitat Politècnica de València, Instituto ITACA, Edif. 8G

Camino de Vera s/n, 46022-València (Spain)

sanjiser@upv.es 